\title{
Dexamethasone Intravitreal Implant
}

National Cancer Institute

\section{Source}

National Cancer Institute. Dexamethasone Intravitreal Implant. NCI Thesaurus. Code C99171.

An intravitreal implant containing the corticosteroid dexamethasone embedded in a biodegradable polymer matrix, with anti-inflammatory and macular edema relieving activity. Upon insertion into the vitreous cavity, dexamethasone intravitreal implant is dissolved slowly and dexamethasone is released over an extended period of time.

Dexamethasone inhibits inflammation thereby preventing leakage from the capillaries and a reduction of retinal edema. This may ultimately prevent vision impairment. 